\title{
CONCURRENT ORDER IN A SEMI-CRYSTALLINE DIBLOCK COPOLYMER INVOLVING COMPLEXATION WITH A MESOGEN
}

Jingbo Wang ${ }^{1}$, Yaroslav I. Odarchenko ${ }^{2}$, Matthieu Defaux ${ }^{1}$, Janis Lejnieks ${ }^{1}$, Denis Ahokhin ${ }^{3}$, Helmut Keul ${ }^{1}$, Dimitri A. Ivanov ${ }^{2,3,{ }^{*},}$, Martin Möller ${ }^{1}$, Ahmed Mourran ${ }^{1, *}$

${ }^{1}$ Interactive Materials Research DWI at RWTH Aachen University and Institut für Technische und Makromolekulare Chemie der RWTH Aachen University, Forckenbeckstr. 50, D-52056 Aachen, Germany;

${ }^{2}$ Institut de Sciences des Matériaux de Mulhouse-IS2M, CNRS UMR7361, Jean Starcky, 15, F-68057 Mulhouse, France;

${ }^{3}$ Moscow State University, Faculty of Fundamental Physical and Chemical Engineering, 119991, Moscow, Russia.

Abstract. A semicrystalline / liquid-crystalline diblock copolymer formed by complexation of a wedge-shaped ligand, 4'-(3', 4',,5'-tris(octyloxy) benzamido) propanoic acid, with P2VPPEO copolymer was studied. The P2VP-complex forms layers with a liquid-crystalline (LC) order causing phase segregation of the PEO block. The molar ratio ligand/pyridine (x) determines the strength of segregation between the blocks and the microphase morphology. For $x \geq 0.5$, PEO forms cylinders within the LC-matrix. The phase separation strongly shifts the crystallization temperature to lower values and forces PEO to crystallize within the block copolymer cylinders. In thin films, alignment of the smectic layers parallel to the substrate induces homeotropic orientation of the PEO cylinders. Inside these cylinders, the crystalline stems preferentially orient along the smectic normal. For $x \leq 0.33$, PEO forms crystalline lamellae within the LC-matrix and crystallization dominates the final structure. 


\section{Graphical Abstract}

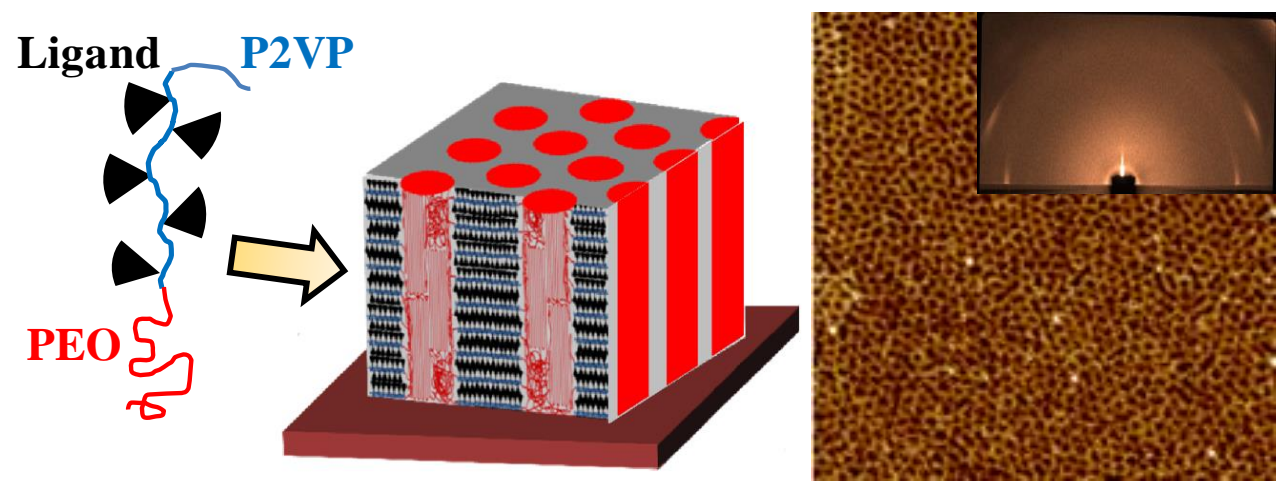

\section{Introduction}

The continuing interest in block copolymers can be accounted for their unique capacity to form a wealth of microphase-separated morphologies, which are of potential interest for the growing field of nanotechnology_: ${ }^{1}$ For instance, block copolymer lithography enabled cost effective fabrication of devices such as flash memory, nanowire transistors, gas sensors and patterned magnetic recording media etc. For the-diblock copolymers with a crystallizable block the resulting structure depends not only on the block composition and segregation strength, but also on the complex interplay between the crystallization behavior of the semicrystalline block and the glass transition of the amorphous block. ${ }^{2-9}$ Two confinement regimes with different crystallization mechanisms are observed, the so-called hard and soft confinement. ${ }^{4}, 9,10$ Thus, the crystallization can remain confined within the microphase separated regions either when the amorphous segment is glassy $\left(T_{\mathrm{ODT}}>T_{\mathrm{g}}>T_{\mathrm{c}}\right)$ or for strongly microphase-separated blocks" (large values of $\chi$ ). This situation is termed "hard confinement regime". In contrast, if the amorphous block is in the rubbery state at the crystallization temperature $\left(T_{\mathrm{ODT}}>T_{\mathrm{c}}>T_{\mathrm{g}}\right)$ or if the driving force of the microphase separation is weak, the diblock copolymer structure can be ruptured by the crystallization process resulting in the so-called "break-out crystallization". ${ }^{11}$ The latter case is termed "soft confinement regime". In general, various nanoconfined environments (such as spheres, ${ }^{8}$ cylinders, ${ }^{7,8}$ and lamellae ${ }^{3,5}$ ) can be generated by the microphase separation of diblock copolymers; these morphologies can be associated to heterogeneous or homogeneous nucleation mechanisms. 
In the liquid-crystalline (LC)-semicrystalline block copolymers, hierarchically-ordered structures can form, with the characteristic scale of the LC ordering being between 1 and 10 $\mathrm{nm}$ and that of the microphase separated domains between 10 and $100 \mathrm{~nm}$. It is expected that crystallization of the crystallizable block can be confined by the mesomorphic matrix. Recently, Zhou et al. showed that the crystallization behavior of a semicrystalline block such as PEO was controlled by the weight fraction of the LC segment in the block copolymer. ${ }^{9}$ Thus if the LC weight fraction is less than 50\%, PEO crystallizes in the "LC lamellae in PEO lamellae" structure at normal undercoolings, while for high LC contents (LC>50\%) PEO crystallizes only at very large undercoolings and forms the "PEO cylinders in LC matrix" structure.

The LC ordering can be introduced via covalent bonding ${ }^{12}$ or via secondary interactions (i.e. hydrogen bonds, ionic interactions, metal ligation) to form a block with comb-like polymers. supramolecular self assembly ${ }^{13-15}$. In the latter case, the mesogen is selectively complexed with one segment of the block copolymer to render it liquid crystalline through non covalent bonding such as ionic interactions, hydrogen bonding or charge transfer interactions. ${ }^{13-18}$ The mesostructures based on ionic interactions are typically thermally stable and in some instances the complexes decompose before reaching the isotropic phase. ${ }^{19-22}$ Recently, we have complexed poly (2-vinylpyridine) (P2VP) with a wedge-shaped ligand consisting of a sulfonate group at the tip and a large non-polar body. In this situation, the final morphology of the system can be influenced by the shape of the mesogen and degree of complexation. $^{20,22}$ In addition, incorporation of such a ligand in a miscible PEO-b-P2VP diblock copolymer ${ }^{23}$ promotes the phase segregation between the blocks. ${ }^{24}$

The complexes based on hydrogen bonding are particularly interesting since this interaction is thermally reversible, selective and directional. ${ }^{13,14}$ In the present paper, we focus on the P2VP-PEO block copolymer complex with a new ligand, 4'-(3", $4^{\prime \prime}, 5^{\prime \prime}$-tris(octyloxy) benzamido) propanoic acid. The structure and thermotropic transitions of some molecules of this homologous series were recently reported. ${ }^{25}$ Here, the bulk structures of the complex with both P2VP homopolymer and P2VP-containing block copolymer have been investigated. The complexation is found to depress the $T_{\mathrm{g}}$ of the glassy block and induce formation of a LC phase, which in turn confines the PEO crystallization and results in crystal orientation in thin films. Also, the segregation strength between the blocks varies with the complexation degree, the fact that provides a possibility to control the morphology and orientation of the monodomains in the thin films. These findings open new opportunities for tuning the crystallinity 
and crystal orientation, which is crucial for several practical applications such as solid-state electrolytes for lithium batteries. ${ }^{26}$

\section{Experimental section}

Materials. $\mathrm{P} 2 \mathrm{VP}_{350}$ homopolymer $\left(\mathrm{M}_{\mathrm{n}}=35000, M_{w} / M_{n}=1.07\right)$ was purchased from SigmaAldrich, diblock copolymer $\mathrm{P}_{2} \mathrm{VP}_{150}-\mathrm{PEO}_{550}$ with $M_{w} / M_{n}=1.18$ was synthesized by sequential anionic polymerization. ${ }^{24}$ The 4 '-(3", 4 ", $5^{\prime \prime}$-tris(octyloxy) benzamido) propanoic acid (C8-Ala$\mathrm{COOH}$, Scheme $\mathrm{S} 1$ ) was used as a ligand and is denoted as $\underline{\mathbf{L}}$. The details of the synthesis were published elsewhere. ${ }^{25}$ Silicon wafers (100) were purchased from CrysTec GmbH. Chloroform (p.a.) and isopropanol (p.a.) were supplied by VWR International and used as received.

Preparation of Polymer Complex. The preparation procedure of block copolymer/ligand complex or homopolymer/ligand complex is similar to the one reported in the literature. ${ }^{24} \underline{I n}$ brief, the $\mathrm{BCP}$ and the ligand were dissolved in chloroform and stirred overnight in the dark. Subsequently, the solvent was evaporated by rotary evaporation under reduced pressure. The solid was collected, re-dissolved in chloroform, and filtered with a $0.2 \mu \mathrm{m}$ PTFE syringe filter. The final concentration of the solutions was controlled to $0.5 \mathrm{wt} \%$ and stored in brown $\mathrm{GC}$ vials. Hydrogen bonding between the $\mathrm{BCP}$ and the ligand was assessed with infrared spectroscopy, see FTIR analysis. All solvents used were of analytical grade and have been bought from VWR GmbH, Germany. Silicon wafers (100) were purchased from Si-Mat $\underline{\mathrm{GmbH}}$. The complexes are denoted as $\mathrm{P} 2 \mathrm{VP}(\underline{\mathbf{L}})_{x}$ and $\mathrm{P} 2 \mathrm{VP}(\underline{\mathbf{L}})_{x}-\mathrm{PEO}$ for the homopolymer/ligand and block copolymer/ligand, respectively, where $x$ is the degree of complexation, defined as the molar ratio of the ligand $(\underline{\mathbf{L}})$ and pyridine moieties.

Film Preparation. Silicon wafers having a ca. $2 \mathrm{~nm}$ thick native silicon oxide layer are used as substrates. Before experiments, they were cleaned by sonication in isopropanol for 5 minutes, followed by drying in $\mathrm{N}_{2}$ stream, and activated with $\mathrm{UV} / \mathrm{O}_{2}$ for 12 minutes. All the substrates were used immediately after the $\mathrm{UV} / \mathrm{O}_{2}$ treatment. The polymer complex was dissolved in chloroform at concentration of $3 \mathrm{wt} \%$. The solution was spun cast (Convac $1001 \mathrm{~S}$, Germany) onto freshly prepared silicon wafers at a speed of $2500 \mathrm{rpm}$ for $30 \mathrm{~s}$ to result in film thickness of ca. $220 \mathrm{~nm}$. The film thickness was measured by ellipsometry (MM-SPEL-VIS, OMT GmbH). Before spin-coating, the solutions were filtered through 0.2 $\mu \mathrm{m}$ PTFE syringe filters. The films were annealed under vacuum $\left(2 \times 10^{-2} \mathrm{mbar}\right)$ at $100^{\circ} \mathrm{C}$ (i.e., 
below the isotropic temperature of the complex) for different times. Subsequently, they were quenched to ambient temperature by quickly transferring the substrates onto a steel plate.

Fourier Transform Infrared Spectroscopy (FTIR) spectra with a resolution of $4 \mathrm{~cm}^{-1}$ were recorded with a Nicolet NEXUS 670 Fourier Transform IR spectrometer. The samples were prepared by drying several droplets of $3 \mathrm{wt} \%$ solution on $\mathrm{KBr}$ plates at room temperature. For each spectrum more than 200 scans were acquired to enhance the signal-tonoise ratio.

Differential Scanning Calorimetry (DSC) measurements were performed on a Netsch DSC 404 instrument under $\mathrm{N}_{2}$ stream. The instrument was calibrated with pure indium. For measurements, about $3 \mathrm{mg}$ of the studied complex was sealed in aluminum pans. The samples were heated to $150^{\circ} \mathrm{C}$, kept at that temperature for 2 minutes to erase the thermal history and then cooled to $-50^{\circ} \mathrm{C}$ at a rate of $10^{\circ} \mathrm{C} / \mathrm{min}$. Subsequently they were heated to $150^{\circ} \mathrm{C}$ at a rate of $10^{\circ} \mathrm{C} / \mathrm{min}$ to analyze the melting behavior. The PEO crystallinity was calculated according to the following equation:

$$
C r=\frac{\Delta H_{f}}{w \cdot \Delta H_{0}} \times 100 \%
$$

where $\Delta H^{f}$ is the measured heat of fusion per one gram of sample, $w$ is the weight percentage of PEO in the block copolymer complex and $\Delta H_{0}$ is the thermodynamic heat of fusion per gram of completely crystalline PEO, which equals $203 \mathrm{~J} / \mathrm{g} .{ }^{27}$

Small- and Wide-Angle X-ray Scattering (SAXS/WAXS) experiments were carried out using a custom-built SAXS/WAXS machine (Molecular Metrology Ltd.) coupled to a Rigaku MicroMax-007HF rotating anode generator. The 2D WAXS data were collected in vacuum using Fuji image plates with a pixel size of $(100 \times 100) \mu \mathrm{m}^{2}$. The modulus of the scattering vector $\mathbf{s}(\mathrm{s}=2 \sin \theta / \lambda$, where $\theta$ is the Bragg angle and $\lambda$ - the wavelength of the photons) was calibrated using several diffraction orders of Ag behenate. WAXS measurements were performed in transmission on oriented samples (fibers) prepared by extruding the polymer complexes with a home-made mini-extruder.

Grazing Incidence X-ray Scattering (GIWAXS) measurements were conducted at the X6B beamline of the National Synchrotron Light Source at Brookhaven National Laboratory using $\lambda$ of $0.79 \AA$. The samples were positioned in environmental chamber isolated with Kapton windows and equipped with a computer-controlled heating stage Instec HCS402 containing a cryo-unit. The focused beam of $0.25 \mathrm{~mm}$ vertical $\times 0.5 \mathrm{~mm}$ horizontal size was directed on the sample at an angle $\theta_{\text {inc }}$ of $0.2^{\circ}$. The diffraction pattern was collected using a 
CCD detector from Princeton Instruments having a $120 \mathrm{~mm} \times 120 \mathrm{~mm}$ image area $(2084 \times$ 2084 pixels). The sample-to-detector distance, typically of $240 \mathrm{~mm}$, was calibrated using Ag behenate powder.

Grazing Incidence Small-Angle X-ray Scattering (GISAXS) experiments were performed on the BW4 beamline at HASYLAB (DORIS storage ring, DESY, Hamburg, Germany). Diffraction patterns were collected with a MAR CCD camera of 2048 x 2048 pixels with a resolution of $79.1 \mu \mathrm{m}$. The wavelength of $1.38 \AA$ and the sample-to-detector distance of $2.31 \mathrm{~m}$ were used in our experiments. The films were measured at incidence angles $\alpha_{i}=0.2$ and 0.3 with typical acquisition time of 200s. The direction of the scattered intensity can be described with the help of two angles: $\alpha_{f}$ (measured in the plane normal to the film surface) and $2 \theta_{f}$ (measured in the film plane). The modulus of the scattering vector $\mathbf{s}$ was calculated as $s=\sqrt{s_{y}^{2}+s_{z}^{2}}$ where $s_{y}=\cos \left(\alpha_{f}\right) \sin \left(2 \theta_{f}\right) / \lambda$ and $s_{z}=\left[\sin \left(\alpha_{f}\right)+\right.$ $\left.\sin \left(\alpha_{i}\right)\right] / \lambda$.

Scanning Force Microscope (SFM). The morphology of the polymer complex films was investigated under ambient conditions in Tapping Mode with a NanoScope IIIa instrument (Digital Instruments/ Veeco Metrology, Santa Barbara, CA). Commercially available standard silicon cantilevers (PPP-SEIH-W from Nanosensors) with a spring constant of 5-37 N/m and oscillation frequency of $\sim 125 \mathrm{kHz}$ were used. Both the topography and phase images were recorded; the micrographs were processed with Digital Instruments software, NanoScope, version 5.12r5. The phase images of polymer complex film at high temperature were acquired using a heating stage controlled by a MMHTRS High Temperature Heater Controller (Digital Instruments, Veeco Metrology, Santa Barbara, CA).

The average diameter of the PEO cylinders was determined from SFM phase images. Using standard Nanoscope image analysis software, we can estimate the surface fraction and average radius using height or phase histogram of a given image. I would-delete this) The eaption to the figure is missing! Should it be Figure 1? The next figure number should be then 2 and soon.

Standard polarized optical microscope (POM) Zeiss axioplan was used to analyse the optical birefringence of the samples in reflection.

\section{Results}

FTIR analysis of the block copolymer complex.

Formatted: Font: Bold Formatted: Font: Bold, Font color: Red 
A spectroscopic investigation was undertaken to understand whether the hydrogen bonding between the pyridine group of the polymer backbone and carboxylic group of the ligand is strong enough to induce the complex formation. Previously, Zhu et al. studied the complex formation of P2VP and (4'-[3", 4" ,5" -tris(octyloxy)benzoyloxy]azobenzene-4-sulfonic acid). ${ }^{20}$ However, in that system there was a strong ionic interaction between sulfonic acid and pyridine, whereas the strength of hydrogen bonding in the pyridine/carboxylic group pair, which is in the focus of the present study, is at least three times weaker. ${ }^{28}$
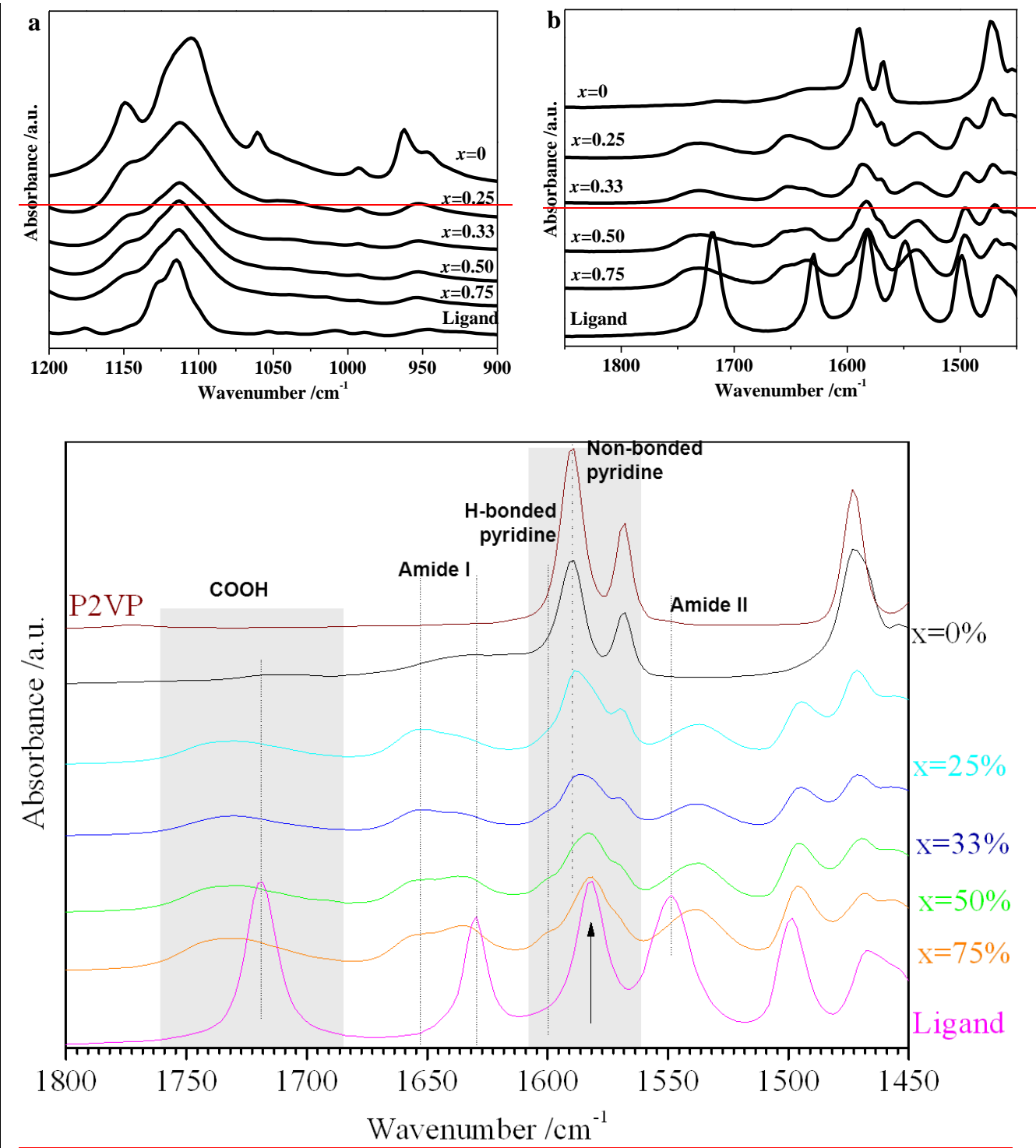


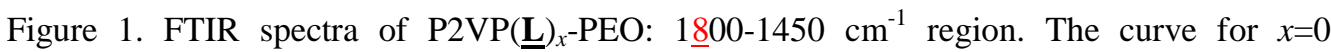
corresponds to the pure copolymer. For the sake of comparison, purpose the FTIR curve of the-P2VP is also added.

Figure 1 shows FTIR spectra of the-copolymers with different complexation degree $x$. The hydrogen bonding interactions between the alanine--based ligand and P2VP can be identified by examining the amide, pyridine and ether regions in the spectra. The ether bands at 1360 $\mathrm{cm}^{-1}$ and $1343 \mathrm{~cm}^{-1}$ are, however, difficult to analyze due to overlapping with the adsorption bands of the ligand. It is noteworthy that the characteristic band of the ether groups remains unaltered regardless of the degree of complexation. WeTherefore, the pyridine and amide bands will be the only ones that are informative for this analysis. are left with the pyridine and the amide banThe two main regions of the FTIR spectra that concern the hydrogen bonding of the ligand to P2VP are shown in Figure 1. The lower wave number band at $1575-1615 \mathrm{~cm}^{-1}$ are associated with the pyridine rings only. ${ }^{129}$ The absorption peak at $1589 \mathrm{~cm}^{-1}$ is due to non-hydrogen bonded pyridine. Close to this band, a shoulder at $1598 \mathrm{~cm}^{-1}$ evolves upon addition of the ligand. This bandIt is due to H-bonded pyridine, and the shoulder indicates thatit shows a relatively a relatively small amountintensity in comparison towith the band of the non-H-bonded pyridine. The spectral change suggests that hydrogen bonding interaction exists between pendant pyridine groups of the P2VP and the ligand. Unfortunately, this spectral region is dominated by signals of phenyl vibration of the ligand, at around $1581 \mathrm{~cm}^{-1}$, which makes any quantitative analysis difficult. ${ }^{30}$

$\underline{\text { Regarding the ligand, a band at } 1719 \mathrm{~cm}^{-1} \text { is associated with the carboxylic acid group which }}$ becomes broad and shifts to a higher wave number (ca. $1730 \mathrm{~cm}^{-1}$ ) in theupon complexationes. The shift in peak position and broadening of the carbonyl region of the spectra may suggest destruction of the acid dimers on association with pyridineduring complexation. However, because of the amide group, H-bonding may involve one or more partners in a complex. Indeed, in the region of the so--called amide I band $1600-1680 \mathrm{~cm}^{-1}$, the pure ligand shows a strong sharp absorption band at $1630 \mathrm{~cm}^{-1}$, presence of the P2VP, induce shifts to higher wavenumber with splits to two broad shoulder, one is around $1654 \mathrm{~cm}^{-}$ 1 , the second is around $1636 \mathrm{~cm}^{-1}$. For higher degree of neutralization, the intensity of the band at $1654 \mathrm{~cm}^{-1}$ decreaseds while the one at $1636 \mathrm{~cm}^{-1}$ increases. The relative intensity of the-two peaks hasve been attributed to the presence of hydrogen--bonded and non-hydrogenbonded amide. In passing we also noteIt is worth noting the changes of the spectral region of the-amide II spectral region where the peak is shifted to lower wavenumbers. All these 
changes indicate that P2VP modifiesy hydrogen bond motifs of the ligand which may account for the observed mesomorphic behavior of the complex as will be discussed later in the text. However a detailed discussion on thatthis issue is beyond the scoepe of the manuscript. To conclude, on mixing, the IR spectruma of the ligand and the-P2VP shows significant changes compared to the spectrumthat of the pure compounds. These changes indicate the presence of $\underline{\text { a strong hydrogen bonding between the acid and pyridine groups. However, tThe spectra are }}$ too complex to allow for a quantitative estimate of the ligand bonded to the P2VP and of the free acid. ${ }^{18,30}$

\section{Bulk structure}

Mesomorphic structure of the homopolymer complex P2VP(L $)_{x}$. Depending on the sample thermal history, the ligand in the pure state reveals two crystalline polymorphs, monoclinic and hexagonal. ${ }^{31}$ The details of the structural analysis of the pure ligand are given in the Supporting Information. It should be emphasized that no LC- state was detected for the ligand. The complexation with P2VP homopolymer changes the ligand structure. Thus, two orders of the fundamental WAXS peak of the complex are observed for all the compositions, thereby showing the formation of a smectic structure upon complexation (cf. Table 1 and Figures S4). The smectic ordering is present up to $x=0.75$, which is at variance with what has been observed for a similar system previously. ${ }^{20}$ Indeed, one would expect formation of a columnar phase due to the space hindrance imposed on the ligand molecules disposed along the P2VP chain. The absence of a smectic-columnar phase transition supports the hypothesis that at high degrees of complexation the hydrogen bonding is not strong enough to drive all of the ligand to the P2VP backbone. As mentioned above, the free ligand molecules ean bare expected to form a dynamic equilibrium with ligands bound to the polymer ${ }^{20}$ probably as isolated dimers. In this context, it is worth mentioning that extruded fibers of the complexes corresponding to high degrees of complexation show decreasing orientation, the fact that can be also accounted for by the free ligand molecules.

Table 1. Structural properties of the as-prepared fibers of $\mathrm{P} 2 \mathrm{VP}(\underline{\mathbf{L}})_{x}$ and $\mathrm{P} 2 \mathrm{VP}(\underline{\mathbf{L}})_{x}$-PEO.

\begin{tabular}{|c|c|c|c|c|c|}
\hline \multirow{2}{*}{ Samples } & \multicolumn{4}{|c|}{ WAXS of the LC phase } & \multirow{2}{*}{$\begin{array}{l}\text { SAXS } \\
L, \mathrm{~nm}\end{array}$} \\
\hline & & ${ }^{x} d_{001}, \mathrm{~nm}$ & $\Delta, \mathrm{nm}^{-1}$ & $d_{002}, \mathrm{~nm}$ & \\
\hline \multirow{3}{*}{$\mathrm{P} 2 \mathrm{VP}(\underline{\mathbf{L}})_{x}$} & 0.25 & 3.66 & 0.088 & 1.81 & - \\
\hline & 0.33 & 3.48 & 0.081 & 1.92 & - \\
\hline & 0.50 & 3.37 & 0.046 & 1.71 & - \\
\hline
\end{tabular}




\begin{tabular}{|c|c|c|c|c|c|}
\hline & 0.75 & 3.28 & 0.059 & 1.76 & - \\
\hline \multirow{5}{*}{$\mathrm{P} 2 \mathrm{VP}(\underline{\mathbf{L}})_{x}-\mathrm{PEO}$} & 0 & - & - & - & 34 \\
\hline & 0.25 & 3.64 & 0.098 & 1.81 & 36 \\
\hline & 0.33 & 3.47 & 0.094 & 1.83 & 40 \\
\hline & 0.50 & 3.20 & 0.084 & 1.68 & 37 \\
\hline & 0.75 & 3.16 & 0.064 & 1.7 & 36 \\
\hline
\end{tabular}

$\Delta$-half width at half maximum (FWHM) of the 001 peak

Block copolymer complex PEO-P2VP(L $)_{x}$. The LC ordering such as described for the case of $\operatorname{P2VP}(\underline{\mathbf{L}})_{\mathbf{x}}$ is preserved in the PEO-P2VP( $(\underline{\mathbf{L}})_{\mathrm{x}}$ complexes: in the WAXS patterns, two peaks with $d$ spacing ratio of 1:2 are observed. The position of the first peak is similar to that of the homopolymer (cf. Table 1 and Figures S5). The presence of the WAXS peaks pertinent to the unit cell of PEO in the as-prepared fibers shows that in all the complexes the PEO bock is semicrystalline (cf. Figures S5). The crystal orientation can be analyzed from positions on the 2D diffractograms of the characteristic peaks at $0.46 \mathrm{~nm}$ and $0.38 \sim 0.40 \mathrm{~nm}$ of the monoclinic lattice of PEO. These peaks correspond to 120 and $\overline{1}_{32}, 032,112, \overline{2} 12, \overline{1}_{24}, \overline{2}_{04}, 004$ reflections, respectively. ${ }^{2}$ For all degrees of complexation the maximum intensity of the 120 peak is on the equator of the diffractograms, which means that the PEO chains in the crystalline lamellae are preferentially oriented parallel to the drawing direction (see Figures S5).

The SAXS technique was used to address the PEO-P2VP $(\underline{\mathbf{L}})_{\mathrm{x}}$ supramolecular structure. In the pure block copolymer, only one broad SAXS peak at $s=0.029 \mathrm{~nm}^{-1}(d=34 \mathrm{~nm})$ is observed (cf. Figures S6). It is known that the P2VP and PEO blocks are miscible at room temperature. Using the analytical method described by Yeh et al. the "lower critical ordering transition" temperature (LCOT) of the studied copolymer was calculated to be $350^{\circ} \mathrm{C}^{23}$ Therefore, the observed SAXS peak can be ascribed to the electron density contrast between the amorphous regions and the PEO crystals. In the polymer complex, a SAXS peak at a similar position is observed. In order to explore the existence of a microphase separation in the block copolymer complex, SAXS experiments were performed at $100^{\circ} \mathrm{C}$ (i.e. above the melting temperature of PEO). In this case, a much sharper interference maximum with a slightly changed $d$-spacing was observed (cf. Figure S6), which allowed us to conclude that the SAXS intensity at room temperature originates at least in part from the microphase-separated domains. The characteristic distance between the domains, $L$, was calculated from the corresponding 1D SAXS curves (cf. Figure S6 and Table 1). 


\section{Crystallization and melting behavior of the block copolymer complexes.}

Figure 2 shows the DSC curves acquired during cooling and subsequent heating of PEO$\mathrm{P} 2 \mathrm{VP}(\underline{\mathbf{L}})_{x}$. The parameters evaluated from these curves are listed in Table 2. It can be seen that, although crystallization and melting of PEO is observed for all samples, the crystallization temperature region is different. Thus, the neat block copolymer (the weight fraction of PEO is $60 \%$ ) does not exhibit a clear exothermic peak upon cooling, but during subsequent heating it undergoes cold crystallization at $-16.1^{\circ} \mathrm{C}$ (Figure 2). Similar behavior has been previously observed for PEO-P2VP copolymer with various PEO weight fraction. ${ }^{32}$ Incorporating of 0.25 of $\underline{\mathbf{L}}$ drastically changes the crystallization behavior of PEO. Although in this case the weight fraction of PEO decreases to $39 \%$, the sample starts to exhibit on cooling a sharp crystallization peak at $20.5^{\circ} \mathrm{C}$. When $x$ increases to 0.33 , the sample shows two crystallization peaks on cooling, one of which is relatively close to room temperature $\left(13.6^{\circ} \mathrm{C}\right)$ and the other one at $-19.1^{\circ} \mathrm{C}$. Further increase of $x$ to 0.50 and to 0.75 leads to disappearance of the room-temperature exothermic peak, while the low-temperature peak remains. A slight decrease of $T_{\mathrm{m}}$ from 59.3 to $57.0^{\circ} \mathrm{C}$ (Figure 2b) and that of crystallinity from $70 \%$ to $50 \%$ (Table 2) are observed when the degree of complexation increases from 0.25 to

0.75 . As it will be discussed later in the text, this temperature shifts is solely associated with the structure of microdomains as it limits the space available for the crystallization of $\mathrm{PEO}^{33}$.

It is noteworthy that in this temperature range the DSC curves do not reveal transitions of the LC- phase.
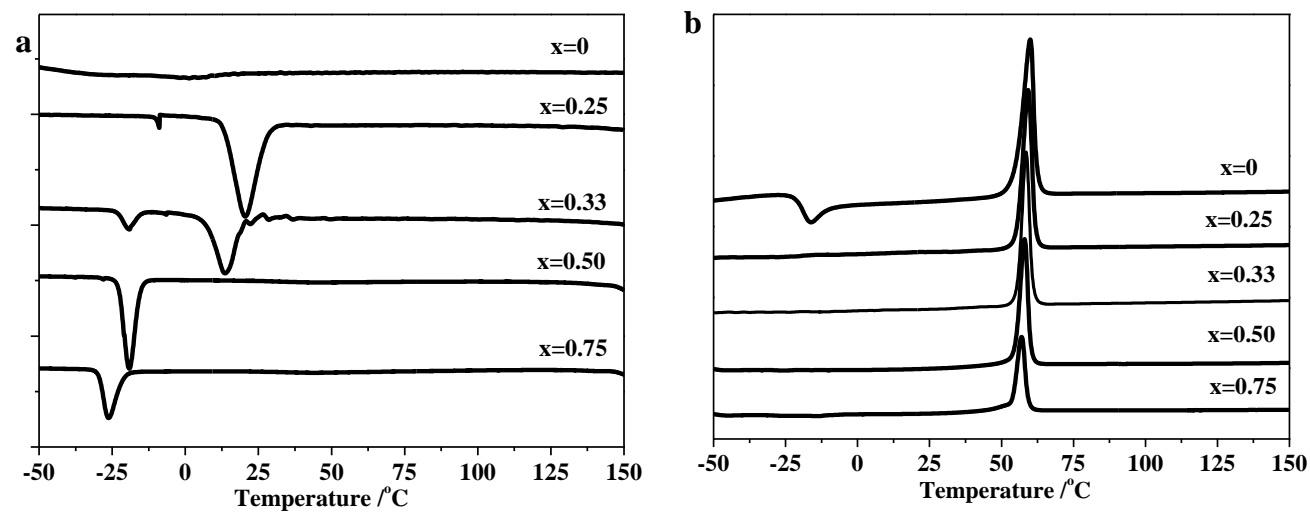

Figure 2. DSC curves of PEO-P2VP( $\underline{\mathbf{L}})_{x}$ with different degree of complexation, $x$, measured during cooling (a) and subsequent heating (b) at $10^{\circ} \mathrm{C} / \mathrm{min}$. 
Table 2. Thermodynamic parameters of $\mathrm{P} 2 \mathrm{VP}(\mathrm{C} 8-\mathrm{Ala}-\mathrm{COOH})_{x}-\mathrm{PEO}$ with different degree of complexation

\begin{tabular}{|c|c|c|c|c|c|}
\hline$x$ & $T_{\mathrm{mp}},{ }^{o} \mathrm{C}$ & $T_{\mathrm{cp}},{ }^{\circ} \mathrm{C}$ & $\Delta T,{ }^{\circ} \mathrm{C}^{*}$ & $H, \mathrm{~J} / \mathrm{g}$ & Crystallinity, \% \\
\hline 0 & -16.1 & 60.0 & 76.1 & 71.5 & 58 \\
\hline 0.25 & 20.5 & 59.3 & 38.8 & 54.7 & 69 \\
\hline 0.33 & $13.6(-19.1)$ & 58.6 & $45.0(77.7)$ & 45.0 & 63 \\
\hline 0.50 & -23.5 & 58.0 & 81.5 & 33.4 & 57 \\
\hline 0.75 & -26.2 & 57.0 & 83.5 & 25.6 & 55 \\
\hline
\end{tabular}

*Supercooling $\Delta T$ is defined as $\Delta T=T_{\mathrm{mp}}-T_{\mathrm{cp}}$

\section{Thin film structure}

The surface morphology was investigated by optical microscopy in combination with SFM. The micro-structural parameters of block copolymer (BCP) morphology are summarized in Table 3. In addition, grazing-incidence X-ray scattering at wide and small angles was performed to assess the structure and the orientation of the crystalline blocks, liquidcrystalline and BCP-microdomains relative to the substrate plane. In general, in the light Tapping Mode conditions, ${ }^{24}$ the SFM images are largely featureless (results not shown here). It is supposed that the hydrophobic alkyl tails of the ligand preferentially adsorb on the film/air interface to reduce the surface energy. This results in a surface layer, which prevents observation of the film microstructure. Since the cantilever can penetrate this surface layer and resolve the microstructures under the hard Tapping Mode conditions, ${ }^{24}$ only the results of hard Tapping Mode are reported here.

Pure PEO-P2VP. Figure 3 displays the SFM and GIWAXS results obtained on pure PEOP2VP block copolymer after thermal annealing. At room temperature, the film surface exhibits densely packed spherulite-like features. Importantly, above the melting point of PEO, SFM does not reveal any signs of the microphase separation, because, as it is mentioned, the temperature employed in the experiments is still far below the LCOT. The 2D GIWAXS pattern (cf. Figure 3b) displays reflections of the monoclinic unit cell of PEO crystals. The position of 120 peak indicates that the crystalline PEO chains are preferentially oriented perpendicular to the substrate. 


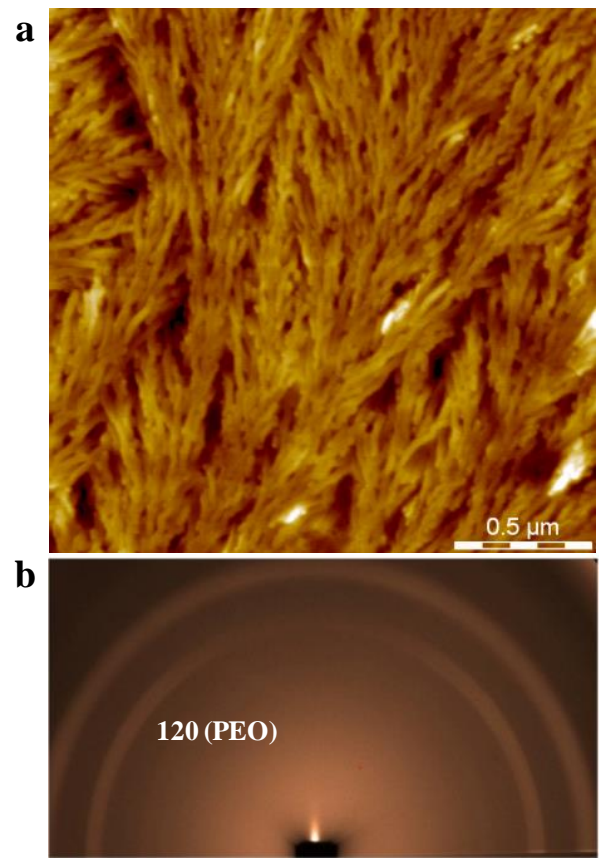

Figure 3. Room-temperature SFM height image (a) and GIWAXS pattern (b) measured on a film of pure P2VP-PEO. The sample was annealed for $24 \mathrm{~h}$ at $100{ }^{\circ} \mathrm{C}$ and subsequently quenched to room temperature.

$\operatorname{PEO-P2VP}(\underline{L})_{0.25}$. At low degree of complexation, the crystallization of PEO leads to formation of a spherulitic texture similar to that observed for the pure block copolymer (cf. Figure 4a). Two halves of the SFM image in Figure $4 \mathrm{~b}$ were recorded at different temperatures. The contrast in the upper half image taken at $50{ }^{\circ} \mathrm{C}$ is rather low since both the semicrystalline PEO and liquid-crystalline $\mathrm{P} 2 \mathrm{VP}(\underline{\mathbf{L}})_{0.25}$ domains are hard. Heating the film above the melting point of PEO makes it possible to discriminate the PEO-rich regions from the LC-complex regions. As illustrated in the lower part of Figure $4 \mathrm{~b}$, at $65{ }^{\circ} \mathrm{C}$ bright discontinuous stripes having periodicity of $36 \pm 2 \mathrm{~nm}$ together with featureless domains are observed. At this temperature, all PEO crystals are molten, but the presence of the mesogen preserves the phase separation between the PEO and LC blocks. Melting of PEO makes the PEO-rich regions to become softer than the LC phase and accounts for their darker contrast in the SFM phase images.

The GISAXS pattern shows the main interference peak positioned on the equatorial direction, which is in agreement with the SFM data (cf. Figure $4 b$ ). This peak corresponds to the distance of $34 \mathrm{~nm}$ and was assigned to 10 reflection generated by the vertically standing 
BCP lamellae. Based on the GIWAXS data (cf. Figure 4d), both the PEO crystals and LCphase are relatively well oriented. The PEO stemserystals have qualitatively the same orientation as the films of pure PEO-P2VP, but the quality of the erystalline-crystals stem erientation is noticeably betteralignment is significantly improved. In this case, the FWHM value of the azimuthal intensity profile of 120 peak is reduced by a factor of two (Fig. S7). Two meridional peaks of the LC-phase with a $d$-spacings ratio of 1:2 are observed, suggesting in agreement with the presence of a smectic phase. The characteristic spacing of the smectic phase is close to the one measured on the bulk samples.
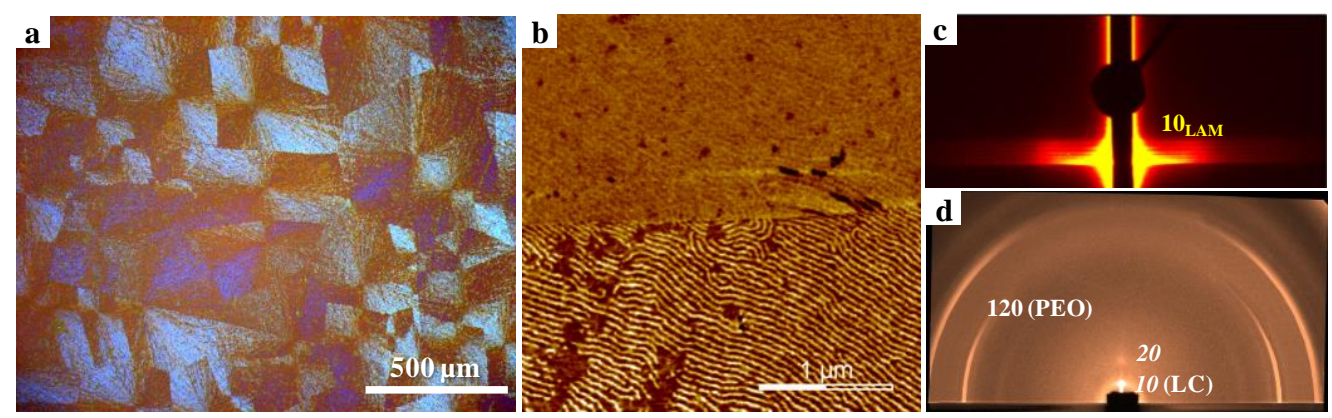

Figure 4. Room-temperature POM image (a), SFM phase image (b) and room-temperature GISAXS (c) and GIWAXS (d) patterns corresponding to a film of PEO-P2VP( $\underline{\mathbf{L}})_{0.25}$. Before measurements, the film was annealed for $24 \mathrm{~h}$ at $100{ }^{\circ} \mathrm{C}$ and subsequently quenched to room temperature. The SFM image recording was started at $50^{\circ} \mathrm{C}$, then the sample was heated insitu to $65^{\circ} \mathrm{C}$. Melting of PEO leads to an improved contrast in SFM as shown in the lower half of the image in (b). GISAXS pattern corresponds to $\alpha_{\mathrm{i}}$ of $0.3^{\circ}$.

PEO-P2VP $(\underline{L})_{0.33}$. For this composition, the POM images still reveal the spherulitic texture although it is only weakly birefringent as compared with the previous case (cf. Figure 5a). Similar to the case of $x=0.25$, the phase contrast in SFM images (Figure $5 b$ ) is enhanced by heating the sample above the melting point of PEO. The SFM images show typical fingerprint morphology, indicating the presence of vertically-standing BCP lamellae with a period of 35 $\pm 3 \mathrm{~nm}$ (cf. Figure $3 \mathrm{~b}$ and Table 3 ). However, the presence of a cylinder structure lying parallel to the substrate cannot be completely ruled out, as they will have a very similar appearance in SFM images. 

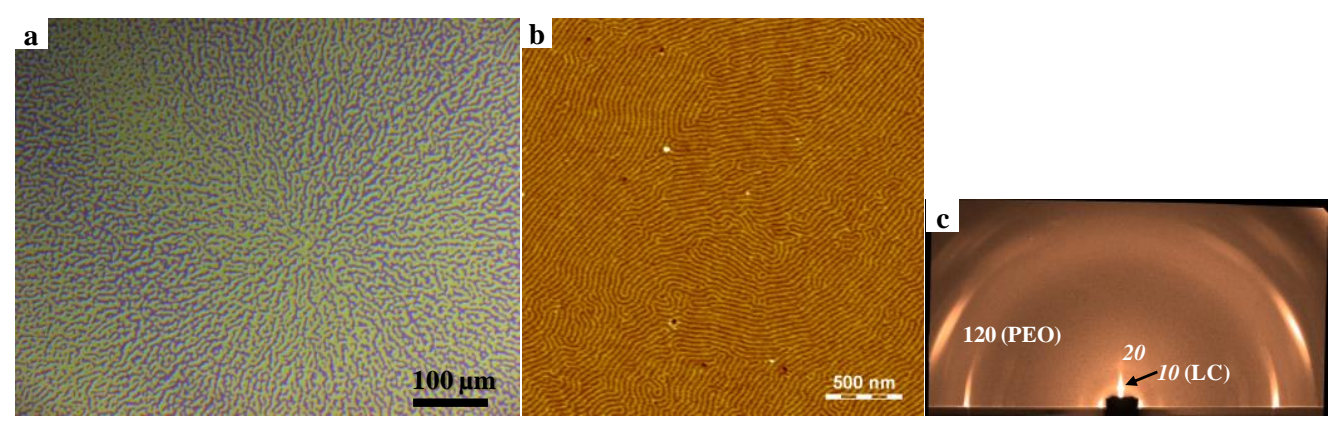

Figure 5. Room-temperature POM image (a), SFM phase image (b) recorded at $65{ }^{\circ} \mathrm{C}$ and room-temperature GIWAXS pattern (c) of a film of PEO-P2VP( $\underline{\mathbf{L}})_{0.33}$. Before measurements, the film was annealed for $24 \mathrm{~h}$ at $100{ }^{\circ} \mathrm{C}$ and subsequently quenched to room temperature.

Indeed, the GISAXS data reveals a mixture of vertically-standing BCP lamellae and lying cylinders (see Fig.6). It is noteworthy that the GISAXS patterns show a superposition of diffracted peaks generated by both the transmitted and reflected X-ray beams (cf. Figure 6). For the incident angle of $0.2^{\circ}$, one observes an equatorial GISAXS peak having the spacing of $35 \mathrm{~nm}$ (denoted as $10_{\text {LAM }}$ in Figure 6a), which corresponds to the vertically-oriented lamellar phase. In addition, a non-equatorial peak (indicated as $10_{\mathrm{HEX}}$ in the same figure) is observed at $30^{\circ}$ with respect to the equator. This peak can be assigned to the hexagonally-packed BCP cylinders lying parallel to the surface. It is noteworthy that in this case one of the $\mathbf{1 0}_{\mathrm{HEX}}$ vectors is directed along the substrate normal (cf. Figure 6a and Figure 8c). By increasing the incident angle, one can change the penetration depth of the X-ray beam. ${ }^{35}$ Since at $0.2^{\circ}$ the incidence angle is very close to the critical angle, only the outer surface is probed. To penetrate deeper in the film, the angle was increased to $0.3^{\circ}$ (Figure $6 \mathrm{~b}$ ). In the corresponding GISAXS pattern, only the equatorial peak is observed, which is in good agreement with the SFM data. This finding shows that the non-equatorial peaks, which are visible for the $\alpha_{\mathrm{i}}$-value of 0.2 , originate exclusively from the outer film layer. By contrast, in the core of the film the BCP morphology mainly consists of vertically-standing lamellae.

The LC ordering of the complex also exhibits both homeotropic and planar orientation, as can be seen from the equatorial and meridional position of the GIWAXS peaks at $3.5 \mathrm{~nm}$ and $1.8 \mathrm{~nm}$. In contrast, the PEO crystals reveal only one orientation with $\boldsymbol{c}$ axis directed along the film normal; the quality of the crystal orientation being improved by about four times as compared to that of $x=0.25$, when judged from the FWHM of the corresponding azimuthal profiles of 120 peak (cf. Fig. S7). as compared to that of $x=0.25$ (Figure 5c). 

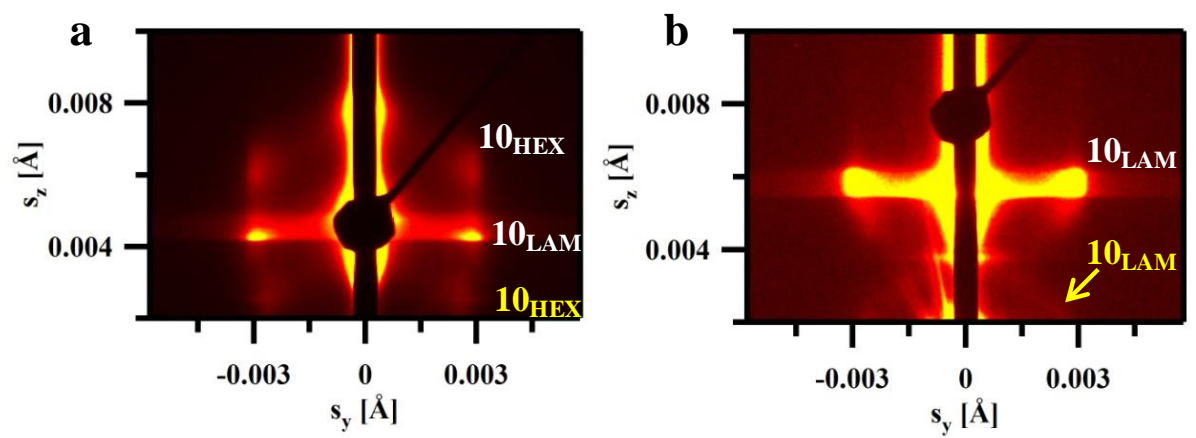

Figure 6. Room-temperature GISAXS patterns of a film of PEO-P2VP( $\underline{\mathbf{L}})_{0.33}$ measured at $\alpha_{\mathrm{i}}=0.2^{\circ}$ (a) and $\alpha_{\mathrm{i}}=0.3^{\circ}$ (b). The scattering peaks generated by the transmitted and reflected beams are given in yellow and white, respectively.

$\operatorname{PEO-P2VP}(\underline{L})_{0.50}$. When the degree of complexation reaches 0.50 , the film is optically isotropic i.e. shows a featureless micrograph (not shown here). Since the weight fraction of PEO decreases in this case to $29 \%$ (cf. Table 3 ), predominantly cylindrical morphology of the PEO block is expected. As shown in Figure 7a, the block copolymer cylinders with periodicity of $39 \pm 4 \mathrm{~nm}$ are well visible with SFM. The phase contrast in the images is already well pronounced at room temperature, which means that crystallinity of PEO block is low. This fact is supported by weaker diffraction from the PEO crystals in GIWAXS (cf. Figure $7 \mathrm{~b}$ ). The GISAXS pattern recorded at the angle $0.2^{\circ}$ displays the peaks, which are separated by $30^{\circ}$ from each other (see Figure 8a). Such pattern indicates on the existence of two types of orientation of lying copolymer cylinders, as schematically illustrated in Figure $8 \mathrm{c}$. When the incident angle is increased to $0.3^{\circ}$ only one equatorial peak remains. This peak having the spacing of $36 \mathrm{~nm}$ (see Figure $8 \mathrm{~b}$ ) can be assigned to 10 reflection of hexagonal phase with homeotropically-oriented cylinders. This finding is in line with the SFM observations. The orientation of the LC phase can be appreciated from the two orders of the fundamental meridional peak of the smectic phase with the characteristic distance of $3.2 \mathrm{~nm}$ (cf. inset of Figure 7b). In this case, the smectic LC layers are parallel to the substrate. Also, highly oriented peaks of the PEO crystals are observed in GIWAXS. The direction of the PEO stems is the same as for lower $x$ values, i.e. perpendicular to the substrate. 


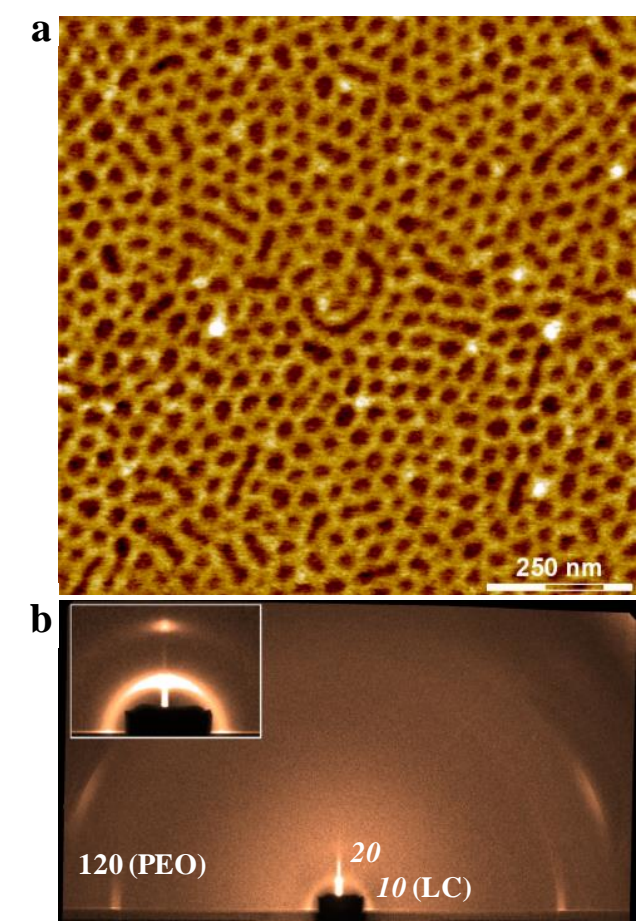

Figure 7. Room-temperature SFM phase image (a) and GIWAXS pattern (b) of PEO-P2VP( $\underline{\mathbf{L}}$ )$_{0.50}$ film. The sample was annealed for $24 \mathrm{~h}$ at $100{ }^{\circ} \mathrm{C}$ and subsequently quenched to room temperature.
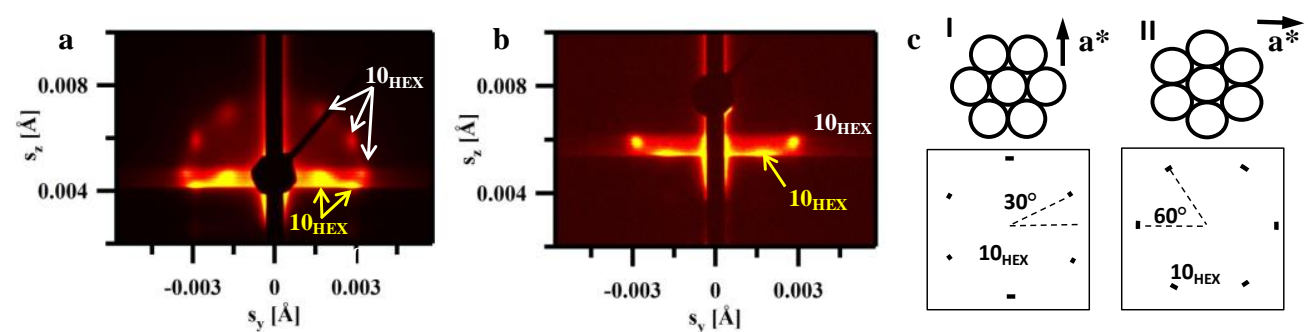

Figure 8. Room temperature GISAXS patterns recorded at $\alpha_{i}=0.2^{\circ}$ (a) and at $0.3^{\circ} \mathrm{C}$ (b) for a film of PEO-P2VP($\underline{\mathbf{L}})_{0.50}$. The scattering peaks generated by the transmitted and reflected beams is given in yellow and white, respectively. Two possible orientations (I) and (II) of the hexagonally-packed cylinders oriented parallel to the surface and the corresponding schematic 2D diffraction patterns (c). 
$\operatorname{P2VP}(\underline{L})_{0.75}$-PEO. At this highest degree of complexation, the weight fraction of PEO is further decreased to $23 \%$. The SFM images exhibit a regular structure of dark dots (Figure 9a). Such morphology with periodicity of $35 \pm 3 \mathrm{~nm}$ can be assigned to PEO cylinders oriented perpendicular to the surface. The absence of GIWAXS peaks prompts us concluding that crystallization is largely suppressed in the sample (see Figure 9b). By contrast, the morphology of the LC phase does not change as compared to $x=0.50$.

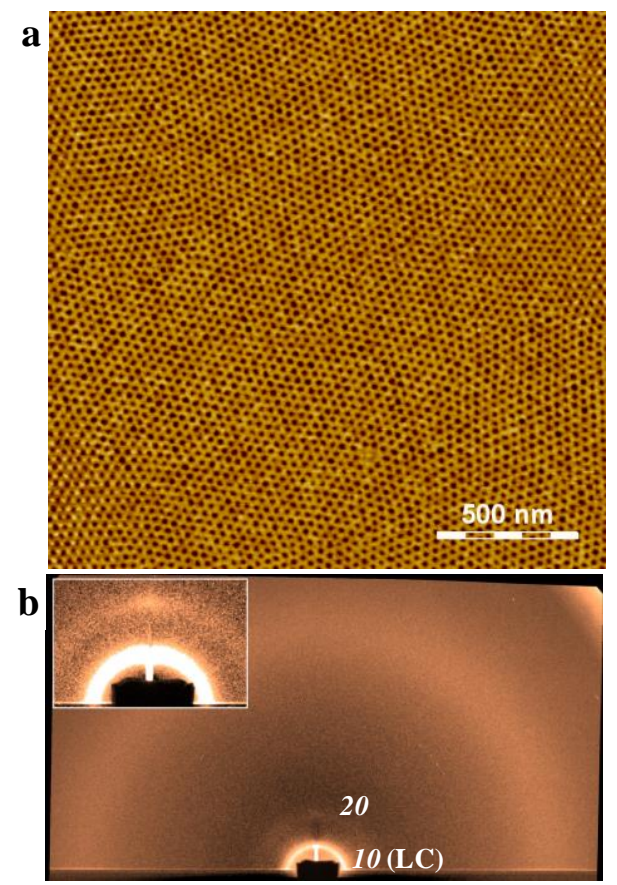

Figure 9. Room-temperature SFM phase image (a) and GIWAXS pattern (b) of PEO$\mathrm{P} 2 \mathrm{VP}(\underline{\mathbf{L}})_{0.75}$ film. The sample was annealed for $24 \mathrm{~h}$ at $100{ }^{\circ} \mathrm{C}$ and subsequently quenched to room temperature.

Table 3. Micro-structural parameters of $\mathrm{PEO}_{560}-\mathrm{P}_{2} \mathrm{VP}_{150}(\underline{\mathbf{L}})_{x}$ films derived from the SFM images

\begin{tabular}{lllll}
\hline & & & $\begin{array}{l}\text { Layer } \\
\text { thickness/ } \\
\text { cylinder } \\
\text { diameter } \\
x\end{array}$ & $\begin{array}{l}w_{\mathrm{LC}}{ }^{*}, \\
d_{\mathrm{PEO}}, \mathrm{nm}\end{array}$ \\
& & Periodicity & Type of morphology \\
& $d_{\text {exp }}, \mathrm{nm}$ & & \\
\hline 0.25 & 61 & $36 \pm 3^{* *}$ & $22 \pm 3$ & Break-out crystallization from
\end{tabular}




\begin{tabular}{|c|c|c|c|c|}
\hline & & & & Sm-in-PEO Lam \\
\hline 0.33 & 65 & $36 \pm 2$ & $22 \pm 3$ & $\begin{array}{l}\text { Sm-in-PEO Lam \& Sm-in-PEO } \\
\text { Cyl (there is also break-out } \\
\text { crystallization here) }\end{array}$ \\
\hline 0.50 & 71 & $39 \pm 4$ & $24 \pm 6$ & $\begin{array}{l}\text { Crystalline PEO Cyl in } \mathrm{Sm} \\
\text { matrix }\end{array}$ \\
\hline 0.75 & 77 & $35 \pm 3$ & $21 \pm 4$ & $\begin{array}{l}\text { Amorphous PEO cylinders in Sm } \\
\text { matrix }\end{array}$ \\
\hline
\end{tabular}

\footnotetext{
${ }^{*}$ Measured from SFM phase images after thermal annealing of the sample for $24 \mathrm{~h}$ at $100^{\circ} \mathrm{C}$

** The spacing was obtained from the power spectral density (PSD) function calculated for the SFM phase image recorded at $65^{\circ} \mathrm{C}$.
}

\section{Discussion}

\section{Liquid-crystalline morphology.}

The phase behavior of a polymer complex depends on a subtle balance between the association interaction and polar-nonpolar repulsion in the ligand/polymer pair. ${ }^{33,36,37}$ Obviously, the hydrogen bonds between the carboxylic groups and pyridine units are energetically more favorable than dimerization of the ligand as evidenced by the red shift of the carbonyl absorption band in the FTIR spectra. The molecular shape of the ligand and phase separation between the P2VP backbone and hydrophobic alkyl tail promote formation of a smectic structure. Similar structure has been reported by Ikkala and co-workers for a simple surfactant $(\mathrm{PDP})^{13,34}$ and a cholesteric derivative. ${ }^{30}$ In principle, one can imagine that the layer-like packing of the mesogens in the complex occurs either with or without interdigitation of the alkyl tails. ${ }^{34}$ In the first case, a smectic layer with a period close to the length of a single mesogen molecule is formed ${ }^{19}$ while the second case brings about a bilayer formation. ${ }^{34}$ For the ligand under study, the high density of alkyls (three alkyl chains per ligand) makes the chain interdigitation unlikely, the fact which is supported by the WAXS results (cf. Figure S4 and Table 1). Indeed, the smectic layer period is about 3.3-3.7 nm, which is close to the double of the molecular length of the ligand in the fully extended state $(\sim 1.7 \mathrm{~nm})$.

The degree of complexation has a noticeable influence on the LC ordering. At low degrees of complexation, the density of the mesogens along the backbone is low, which gives rise to a loosely packed smectic mesophase. Thus, at $x=0.25$, the FWHM of the LC phase fundamental 
peak equals $0.088 \mathrm{~nm}^{-1}$ (cf. Table 1), corresponding to a domain size of about $10 \mathrm{~nm}$, or of approximately three smectic periods. The smectic order improves as more ligand is incorporated: for $x=0.5$ the FWHM is reduced to $0.046 \mathrm{~mm}^{-1}$. Interestingly, the $d$-spacing of the X-ray diffraction peak gradually decreases with increasing $x$, which probably means that higher degrees of complexation result in a more stretched conformation of the P2VP backbone, and, accordingly, in a smaller polymer chain cross-section. Simultaneously, the decrease of FWHM of the LC phase fundamental peak indicates improvement of long range ordering of the smectic phase (cf. Table 1). Noteworthy, the presence of PEO block does not change the type of the mesophase.

\section{Crystallization and orientation of PEO in thin films.}

As mentioned above, PEO and P2VP chains are miscible. It is reported that due to the blocks miscibility PEO cannot crystallize when the weight fraction of the corresponding block is less than $60 \%{ }^{32}$ Introduction of the ligand into the system generates the microphase separation and promotes the LC ordering. We believe that three different confinement regimes are observed in this work: soft confinement at low $x(\leq 0.25)$, co-existence of soft and hard confinements at intermediate $x \sim 0.33$ and hard confinement at higher values of $x(\geq 0.5)$.

Low degrees of complexation. When the sample corresponding to $x=0.25$ is cooled down from the annealing temperature, PEO crystallization occurs at about room temperature, i.e. under normal undercooling conditions $\left(\Delta T=38.8^{\circ} \mathrm{C}\right)$. This undercooling is similar to that of a PEO homopolymer with molecular weight of $30 \mathrm{~kg} / \mathrm{mol}$ (results not shown here). It is likely that at this composition the segregation strength of the microphase separation is not sufficient to efficiently confine the crystallization, and therefore the microphase-separated block copolymer morphology is partially destroyed by the growing PEO crystals. Such crystallization behavior of PEO in the soft confinement is typical for the moderate strength of the microphase separation. ${ }^{11}$ In thin films, the ligand organizes in a smectic phase parallel to the substrate, while the BCP lamellae have vertical orientation. The PEO crystallization proceeds with $\boldsymbol{c}$-axis oriented perpendicular to the substrate. The SFM phase images evidence the existence of microphase-separated regions disturbed by crystallization (cf. Figure $4 a$ ). The schematics of the hierarchical structure of the complex is given in Scheme 1. 


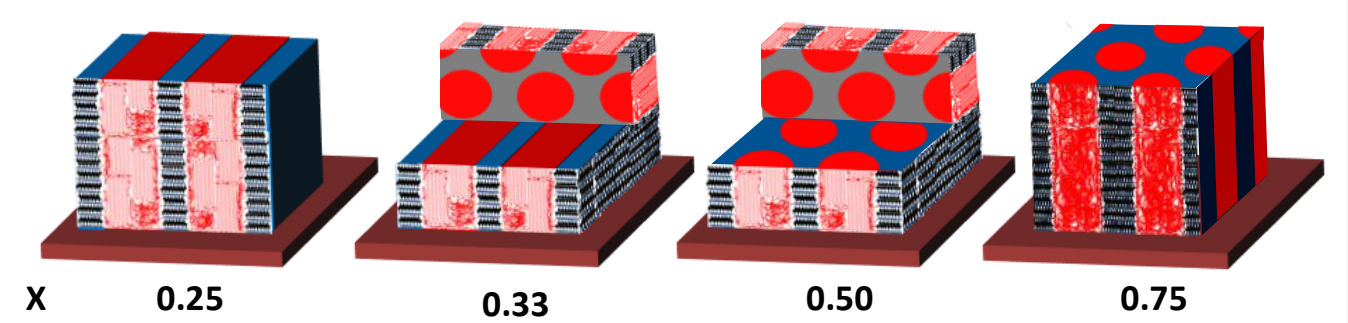

Scheme 1. Schematics of the crystal orientation and LC ordering in thin block copolymer films with different degrees of complexation $(x)$. The heights of the top and bottom layers for $\mathrm{x}=0.33$ and 0.50 are made equal for the sake of clarity. Also, only one orientation of lying $\mathrm{BCP}$ cylinders is depicted for $\mathrm{x}=0.5$ for the same reason.

Intermediate degrees of complexation. The increase of $x$ to 0.33 results in improvement of the LC ordering. In the DSC curves, in additional to the crystallization peak at $\Delta T=45.0^{\circ} \mathrm{C}$ one observes another peak albeit small in the low-temperature region, at $\Delta T=77.7^{\circ} \mathrm{C}$. This undercooling is very close to that of the PEO crystallization in 2D-confined cylinders (e.g., PEO cylinders in PS matrix). ${ }^{35}$ The co-existence of two types of morphologies, i.e. Lam/Cyl is supported by the GISAXS data, with the horizontally-oriented cylinders existing only in the outmost surface layer of the film. Moreover, the horizontal and vertical smectic layers of LCphase observed in GIWAXS (cf. Figure 5c) can be pertinent to the vertically oriented copolymer lamellae and lying cylinders, respectively. In spite of the two morphologies, only one orientation of PEO crystals is observed for which the PEO stems are perpendicular to the film surface, as it is the case for the complex with $x=0.25$ having vertically standing BCP lamellae. One might also expect to see a second population of PEO crystals oriented parallel to the substrate, which are pertinent to the structure of lying cylinders. However, no such orientation is found. One possible reason can be that the fraction of cylinder domains is insufficient to be visible in the GIWAXS patterns. The structural model corresponding to the degree complexation of 0.33 is shown in Scheme 1.

High degrees of complexation. For $x \geq 0.5$, larger undercoolings $\left(\Delta T>80^{\circ} \mathrm{C}\right)$ are necessary for the PEO crystallization, suggesting the hard confinement conditions. ${ }^{9}$ Already at $x=0.50$, a hierarchical morphology of the 2D-confined PEO cylinders immersed in the smectic LC matrix is observed. Narrow azimuthal width of the GIWAXS peaks, which testifies high crystal orientation (Figure 7c) together with less crystalline PEO in comparison with complexes at lower $x$-values, confirms that crystallization is fully confined. Since the PEO 
crystalline lamella thickness is close to the diameter of the PEO domains (Table 3), the preferential crystal growth direction is along the cylinder axis. The schematic model of the cross section of the thin film is shown in Scheme 1. Interestingly, a fraction of copolymer cylinders in the top surface layer of the film is still oriented horizontally but these lying cylinders do not contribute visibly to the overall orientation of the PEO crystals in the film.

If even more ligand is added $(x=0.75)$, the PEO crystallization in thin films becomes largely suppressed. A hierarchical morphology (PEO cylinders in LC matrix) similar to that of $x=0.50$ is observed, with the exception that in this case PEO remains amorphous. A possible explanation of this fact is the reduction of the PEO cylinder diameter (cf. Table 3). This effect is also confirmed by DSC showing large undercooling typical of homogeneously nucleated PEO (Table 2). The structural model for this composition is given in Scheme 1.

To explain the inability of PEO to crystallize one could evoke the possibility of the free ligand ligand to form a complex with oxygen of the PEO ether group thereby perturbing the PEO chain conformation. However, this possibility can be ruled out, since no evidence of hydrogen bonding between the ligand and ether ${ }^{36}$ is detected in the FTIR spectra (cf.

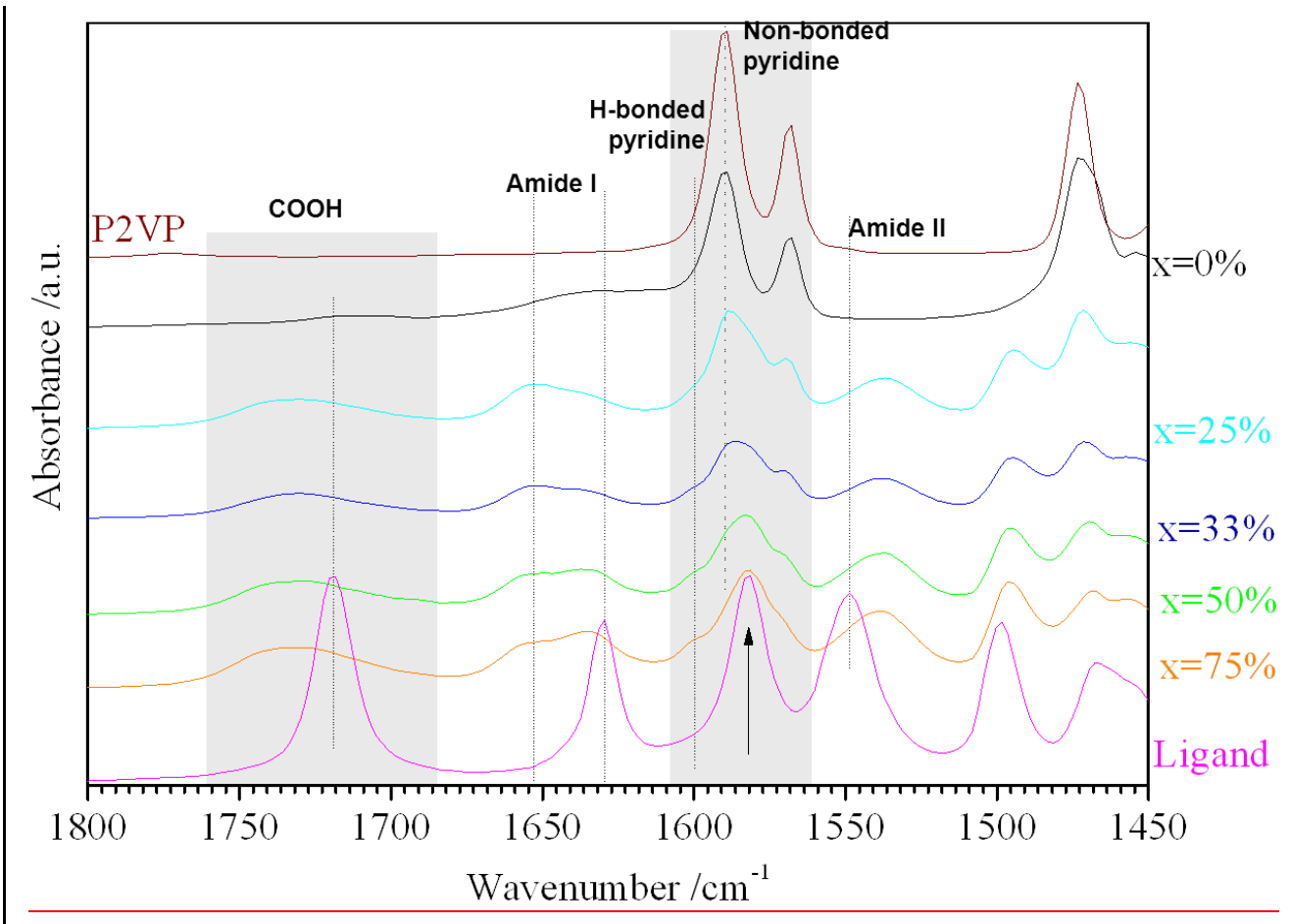

Figure 1). 


\section{Conclusions}

This work describes the formation of a complex between a novel wedge-shaped molecule, $4^{\prime}-\left(3^{\prime \prime}, 4^{\prime \prime}, 5^{\prime \prime}\right.$-tris(octyloxy) benzamido) propanoic acid and a PEO-P2VP block copolymer. Despite the absence of LC-phase for the pure ligand, the complexes with P2VP homopolymer or with PEO-P2VP diblock copolymer exhibit a smectic phase, the ordering degree of which depends on the ligand content. For the block copolymer, the ligand induces a microphase separation between the blocks controlled by the degree of complexation. A transition from the BCP lamellae disrupted by crystallization of PEO through coexistence of lamellae/cylinders to cylinders is consistently observed.

It is found that the LC ordering can confine the PEO crystallization. Different confinement environments can be generated as a function of the degree of complexation, $x$. At low values of $x$ (e.g., $x=0.25$ ), the lamellar BCP-morphology and insufficient segregation strength result in soft confinement for the PEO crystallization, which occurs at normal undercooling. Increasing the $x$-value leads to enhanced LC ordering. This provides hard confinement for crystallization, similar to the glassy polymer matrices. Thus at $x=0.33$, two nanoconfinement environments co-exist such as the PEO lamellae and PEO cylinders in the LC matrix. Accordingly, two undercooling conditions are observed for the PEO crystallization: the "normal" undercooling values for the PEO lamellae (1D) and larger values for the PEO cylinders (2D). The pure 2D confinement is observed when the degree of complexation is further increased to 0.5 . In this case, large undercooling is required for the PEO crystal nucleation since it occurs homogeneously, within isolated cylindrical domains. At the same time, the confinement reduces the maximum degree of crystallinity attainable in thin films, and eventually the crystallization becomes completely depressed at $x=0.75$.

During crystallization in the cylindrical phase at room temperature only the PEO crystals with the stems parallel to the cylinder axis are stable thermodynamically as the pore diameter imposes a strong geometrical constraint on the growing crystals.

\section{Acknowledgements}

A.M and H.K acknowledges financial support from the German Federal Ministry of Education and Research (Nanohybride grant no. 13N9126) and from the European Union (EC Marie Curie ITN project Hierarchy - PITN-CA-2008-215851). GIXD measurements were carried out at the National Synchrotron Light Source, Brookhaven National Laboratory, which is supported by the U.S. Department of Energy, Division of Materials Sciences and Division of Chemical Sciences, under Contract DE-AC02-98CH10886. The authors acknowledge E. 
DiMasi (X6B beamline of the BNL) for the help and excellent experimental environment of the beamline. The authors are grateful to Jan Perlich (BW4 beamline at the DESY) for assistance with GISAXS experiments. D.A. and D.A.I. acknowledge the French Agence Nationale de la Recherche (SPIRWIND and T2T projects) for financial support. Y.O. is a fellow of the Programme INTERREG IV Upper Rhine, Project No.C25 "RHIN-SOLAR". The authors are grateful to the Russian Ministry of Science and Education (grants No $11 \mathrm{G} 34.31 .0055$ and 14.518.11.7013) for financial support.

\section{References}

1. Krikorian, V.; Kang, Y.; Thomas, E. L., Self-Assembly and Morphology Diagrams for Solution and Bulk Materials: Experimental Aspects. In Macromolecular Engineering, WileyVCH Verlag GmbH \& Co. KGaA: 2007; pp 1387-1430.

2. Zhu, L.; Cheng, S. Z. D.; Calhoun, B. H.; Ge, Q.; Quirk, R. P.; Thomas, E. L.; Hsiao, B. S.; Yeh, F.; Lotz, B. J. Am. Chem. Soc. 2000, 122, (25), 5957-5967.

3. Huang, P.; Zhu, L.; Guo, Y.; Ge, Q.; Jing, A. J.; Chen, W. Y.; Quirk, R. P.; Cheng, S. Z. D.; Thomas, E. L.; Lotz, B.; Hsiao, B. S.; Avila-Orta, C. A.; Sics, I. Macromolecules 2004, 37, (10), 3689-3698.

4. Zhu, L.; Mimnaugh, B. R.; Ge, Q.; Quirk, R. P.; Cheng, S. Z. D.; Thomas, E. L.; Lotz, B.; Hsiao, B. S.; Yeh, F.; Liu, L. Polymer 2001, 42, (21), 9121-9131.

5. Sun, Y.-S.; Chung, T.-M.; Li, Y.-J.; Ho, R.-M.; Ko, B.-T.; Jeng, U. S.; Lotz, B. Macromolecules 2006, 39, (17), 5782-5788.

6. Chung, T.-M.; Wang, T.-C.; Ho, R.-M.; Sun, Y.-S.; Ko, B.-T. Macromolecules 2010, 43, (14), 6237-6240.

7. Huang, P.; Guo, Y.; Quirk, R. P.; Ruan, J.; Lotz, B.; Thomas, E. L.; Hsiao, B. S.; Avila-Orta, C. A.; Sics, I.; Cheng, S. Z. D. Polymer 2006, 47, (15), 5457-5466.

8. Chen, H.-L.; Wu, J.-C.; Lin, T.-L.; Lin, J. S. Macromolecules 2001, 34, (20), 69366944.

9. Zhou, Y.; Ahn, S.-k.; Lakhman, R. K.; Gopinadhan, M.; Osuji, C. O.; Kasi, R. M. Macromolecules 2011, 44, (10), 3924-3934.

10. Nandan, B.; Hsu, J. Y.; Chen, H. L. Journal of Macromolecular Science, Part C 2006, 46, (2), 143-172.

11. Xu, J.-T.; Fairclough, J. P. A.; Mai, S.-M.; Ryan, A. J.; Chaibundit, C. Macromolecules 2002, 35, (18), 6937-6945. 
12. Tenneti, K. K.; Chen, X.; Li, C. Y.; Shen, Z.; Wan, X.; Fan, X.; Zhou, Q.-F.; Rong, L.; Hsiao, B. S. Macromolecules 2009, 42, (10), 3510-3517.

13. Ruokolainen, J.; Mäkinen, R.; Torkkeli, M.; Mäkelä, T.; Serimaa, R.; Brinke, G. t.; Ikkala, O. Science 1998, 280, (5363), 557-560.

14. Ikkala, O.; ten Brinke, G. Science 2002, 295, (5564), 2407-2409.

15. Valkama, S.; Ruotsalainen, T.; Nykänen, A.; Laiho, A.; Kosonen, H.; ten Brinke, G.; Ikkala, O.; Ruokolainen, J. Macromolecules 2006, 39, (26), 9327-9336.

16. Chao, C.-Y.; Li, X.; Ober, C. K. Pure Appl. Chem. 2004, 76, 1337.

17. Chen, H.-L.; Lu, J.-S.; Yu, C.-H.; Yeh, C.-L.; Jeng, U. S.; Chen, W.-C.

Macromolecules 2007, 40, (9), 3271-3276.

18. Chuang, W.-T.; Sheu, H.-S.; Jeng, U. S.; Wu, H.-H.; Hong, P.-D.; Lee, J.-J. Chem. Mater. 2009, 21, (6), 975-978.

19. Gopinadhan, M.; Beach, E. S.; Anastas, P. T.; Osuji, C. O. Macromolecules 2010, 43, (16), 6646-6654.

20. Zhu, X.; Beginn, U.; Möller, M.; Gearba, R. I.; Anokhin, D. V.; Ivanov, D. A. Journal of the American Chemical Society 2006, 128, (51), 16928-16937.

21. Ikkala, O.; Ruokolainen, J.; ten Brinke, G.; Torkkeli, M.; Serimaa, R. Macromolecules 1995, 28, (21), 7088-7094.

22. Zhu, X.; Tartsch, B.; Beginn, U.; Möller, M. Chem. - A Eur. J. 2004, 10, (16), 38713878 .

23. Yeh, C.-L.; Hou, T.; Chen, H.-L.; Yeh, L.-Y.; Chiu, F.-C.; Müller, A. J.; Hadjichristidis, N. Macromolecules 2011, 44, (3), 440-443.

24. Albrecht, K.; Mourran, A.; Zhu, X.; Markkula, T.; Groll, J.; Beginn, U.; de Jeu, W. H.; Moeller, M. Macromolecules 2008, 41, (5), 1728-1738.

25. Anokhin, D. V.; Lejnieks, J.; Mourran, A.; Zhu, X.; Keul, H.; Möller, M.; Konovalov, O.; Erina, N.; Ivanov, D. A. ChemPhysChem 2012, 13, (6), 1470-1478.

26. Staunton, E.; Andreev, Y. G.; Bruce, P. G. Faraday Discussions 2007, 134, (0), 143156.

27. Martuscelli, E.; Silvestre, C.; Addonizio, M. L.; Amelino, L. Makromol. Chem. 1986, $187,(6), 1557-1571$.

28. Arnett, E. M.; Ahsan, T.; Amarnath, K. J. Am. Chem. Soc. 1991, 113, (18), 6858-6861.

29. Lee, J. Y.; Painter, P. C.; Coleman, M. M. Macromolecules 1988, 21, (4), 954-960. 
30. Korhonen, J. T.; Verho, T.; Rannou, P.; Ikkala, O. Macromolecules 2010, 43, (3),

Formatted: German (Germany) 1507-1514.

31. Wang, J.; de Jeu, W. H.; Speiser, M.; Kreyes, A.; Ziener, U.; Magerl, D.; Philipp, M.; Muller-Buschbaum, P.; Moller, M.; Mourran, A. Soft Matter 2013, 9, (4), 1337-1343.

32. Córdova, M.; Lorenzo, A. T.; Müller, A. J.; Fragouli, P.; Iatrou, H.; Hadjichristidis, N. Macromol. Symp. 2010, 287, (1), 101-106.

33. Pfefferkorn, D.; Kyeremateng, S. O.; Busse, K.; Kammer, H.-W.; Thurn-Albrecht, T.; Kressler, J. r. Macromolecules 44, (8), 2953-2963.

34. Luyten, M. C.; Alberda van Ekenstein, G. O. R.; ten Brinke, G.; Ruokolainen, J.; Ikkala, O.; Torkkeli, M.; Serimaa, R. Macromolecules 1999, 32, (13), 4404-4410.

35. Huang, P.; Zhu, L.; Cheng, S. Z. D.; Ge, Q.; Quirk, R. P.; Thomas, E. L.; Lotz, B.; Hsiao, B. S.; Liu, L.; Yeh, F. Macromolecules 2001, 34, (19), 6649-6657.

36. Lee, J. W.; Lee, C.; Choi, S. Y.; Kim, S. H. Macromolecules 2009, 43, (1), 442-447. 